\title{
REFLEX SYMPATHETIC DYSTROPHY SECONDARY TO LEPROSY PURE NEURAL FORM
}

Laura Maria Silva de Siqueira1, , Douglas Squizatto Leite ${ }^{1}$, João Flávio Gomes Faria ${ }^{1}$, Daniela Esteves Temporim, Andrea de Almeida Peduti Batista ${ }^{1}$, Sula Glaucia Lage Drumond Pacheco ${ }^{1}$, Sean Hideo Shirata Lanças ${ }^{1}$, Henrique Pereira Sampaio ${ }^{1}$, Luiz Eduardo Valente ${ }^{1}$, Matheus Zanata Brufatto ${ }^{1}$

1. Universidade Estadual Paulista “Júlio de Mesquita Filho", Botucatu (SP), Brazil.

*Corresponding author: mss.laura@yahoo.com

\section{BACKGROUND}

Leprosy is caused by Mycobacterium leprae, a mycobacterium with an affinity for skin cells and peripheral nerves. It represents a public health problem because it causes physical, social, and economic disability. Brazil has not yet reached the eradication by Word Health Organization target.

\section{CASE REPORT}

Male, 57 years old, hypertensive, smoker, alcoholic. Two months ago, he started pain in the heels and dorsum of the feet with heat, edema, sweating, and redness extending to the pretibial region associated with paresthesia and functional limitation. He evolved with pain, heat, redness, edema, sweating, and atrophy of the hypothenar regions and palmar fascia, inflammatory arthralgia and thickening of the distal and proximal interphalangeal, associated with paresthesia and piloerection of the hands. Reflexes: biceps, triceps, styloradials present and symmetricals with normal global motor strength except in the right upper limbs (grade IV) and bilateral ulnar nerve thickening. Absence of right patellar reflex with bilateral quadriceps atrophy. There were no skin lesions on the lower or upper limbs. The hypothesis of early reflex sympathetic dystrophy and neural form leprosy was made. He started $10 \mathrm{mg} / \mathrm{day}$ of prednisone. In bone scintigraphy observed in the wrist and right-hand reflex sympathetic dystrophy in the inflammatory phase. In electroneuromyography presence of peripheral neuropathy mononeuritis multiple with an axonal and demyelinating component, significant etiological differential with leprosy in addition to preganglionic changes superimposed in lower limbs, root, and motor neuron body. Thoracic, lumbar, sacral spine CT scan and unaltered hands radiograph. Hypotheses confirmed, treatment was started with a paucibacillary polychemotherapeutic regimen with $300 \mathrm{mg} / \mathrm{month}$ of rifampicin and $100 \mathrm{mg} / \mathrm{day}$ and $100 \mathrm{mg} / \mathrm{month}$ of dapsone, in addition to $10 \mathrm{mg} /$ day of prednisone. In 3 months, there was an improvement in pain and edema in the hands and feet, maintaining paresthesias. Reassessments continue with a proposal to introduce tricyclic antidepressants, gabapentinoids, and anticonvulsants to address chronic pain as the antimicrobial treatment progresses.

\section{CONCLUSION}

In clinical rheumatologic practice, infections act as triggers for disease development and activity. To perform rheumatological differential diagnoses with infectious diseases taking into account the Brazilian reality, clinical history, and rheumatological physical examination is essential for better treatment and cure of the patient. In this case, the clinical history of neuropathic pain associated with the targeted physical examination with thickening of peripheral nerves was fundamental for clinical suspicion, since no skin lesions were identified.

\section{KEYWORDS}

Reflex sympathetic dystrophy, Leprosy pure neural form, Paresthesias. 